\title{
COMÉRCIO DE FRUTAS CÍTRICAS EM CURITIBA E REGIÃO METROPOLITANA
}

\author{
Trade of citrus in Curitiba and in its metropolitan region
}

\author{
Ricardo Cetnarski Filho ${ }^{1}$, \\ Ruy Inácio Neiva De Carvalbo ${ }^{2}$ \\ Vinícius Caetano Martin ${ }^{3}$
}

\section{Resumo}

O objetivo deste trabalho foi levantar a variação de preços no mercado varejista das frutas cítricas em Curitiba e Região Metropolitana no período de fevereiro de 1999 a janeiro de 2000. Quatro mercados representativos do comércio de frutas na região foram selecionados e visitados mensalmente para coleta de preços praticados. Os preços reais foram corrigidos pelo INPC-IBGE para comparação entre os meses do ano. As cultivares de laranja comercializadas foram a 'Baía', 'Pêra' e 'Lima' com os seguintes preços médios $R \$ 1,18 / \mathrm{kg}, \mathrm{R} \$ 0,41 /$ $\mathrm{kg}$ e $\mathrm{R} \$ 0,87 / \mathrm{kg}$, respectivamente. As cultivares de lima encontradas foram a 'Tahiti' com preço médio de $\mathrm{R} \$$ $0,78 / \mathrm{Kg}$ e a 'Pérsia' com preço médio $\mathrm{R} \$ 1,37 / \mathrm{kg}$. As principais cultivares de tangerina comercializadas foram a 'Ponkan' e 'Murcote'. Foram agrupadas e chamadas de 'outras cultivares' as cultivares 'Paulista', 'Bravo' e 'Rio'. Havia também 'cultivares não identificadas'. O preço médio encontrado no varejo foi de R\$ 0,56/kg para a cultivar 'Ponkan', R \$ 1,19/kg para a cultivar 'Murcote', $\mathrm{R} \$ 0,75 / \mathrm{kg}$ para o grupo das outras cultivares e R\$ $1,29 / \mathrm{kg}$ para as cultivares não identificadas.

Palavras-chave: Mercado, Preço, Laranja, Lima, Tangerina.

\section{Abstract}

The objective of this work was to assess the variation of prices in the market of citrus in Curitiba and its Metropolitan Region from February/1999 to January/2000. The prices were monthly collected in four representatively selected markets. The real prices were corrected by the INPC-IBGE for comparison among the months of the year. The cultivars of orange marketed were 'Baía, 'Pera' and 'Lima' with the average prices of $\mathrm{R} \$ 1,18 / \mathrm{kg}, \mathrm{R} \$ 0,41 / \mathrm{kg}$ and $\mathrm{R} \$ 0,87 / \mathrm{kg}$, respectively. The lime cultivars marketed were 'Tahiti' with average price of $\mathrm{R} \$ 0,78 / \mathrm{kg}$ and 'Persia' with average price of $\mathrm{R} \$ 1,37 / \mathrm{kg}$. The marketed cultivars of tangerine were 'Ponkan', 'Murcote', 'Paulista', 'Bravo', 'Rio' and 'non-identified cultivars'. The cultivates 'Paulista', 'Bravo' and 'Rio' were called 'other cultivate' because the low presence in the different markets. The average price found was $\mathrm{R} \$ 0,56 / \mathrm{kg}$ for 'Ponkan', $\mathrm{R} \$ 1,19 / \mathrm{kg}$ for 'Murcote', $\mathrm{R} \$ 0,75 / \mathrm{kg}$ for the group of other cultivars and $\mathrm{R} \$ 1,29 /$ $\mathrm{kg}$ for non-identified cultivars.

Keywords: Marketing, Price, Orange, Lime, Tangerine.

1 Engenheiro Agrônomo. Mestre. Autor para correspondência. Rua Joinvile, n. 2777, CEP 83020-020. São José dos Pinhais, PR. EMail: ric@floresta.ufpr.br

2 Engenheiro Agrônomo, Dr., Professor Adjunto I da PUCPR, Campus São José dos Pinhais. Rodovia BR 376, km 14. Caixa Postal 129 - CEP 83010-500. São José dos Pinhais, PR. E-Mail: ruycarvalho@zipmail.com.br

3 Engenheiro Agrônomo. Rua Clovis de Vilaqua Sobrinho, n. ${ }^{\circ}$ 284, CEP 81570-170. Curitiba-Pr. E-Mail: vicamar@bol.com.br 


\section{Introdução}

Alguns historiadores afirmam que os cítricos teriam surgido no leste asiático, nas regiões que incluem hoje a Índia, China, Butão, Birmânia e Malásia. Segundo pesquisadores, eles foram levados da Ásia para o norte da África e de lá para o sul da Europa, onde teriam chegado na Idade Média. Da Europa foram trazidos para as Américas na época dos descobrimentos, por volta de 1500 (ABECITRUS, 2001). A primeira espécie a ser conhecida foi a cidra, a seguir a laranja azeda, o limão, a laranja-doce e por último, a tangerina (SIMÃO, 1998).

Dados da FAO (1995), citados por Simão (1998), mostram que o Brasil é um dos principais países produtores das frutas cítricas no mundo, responsável por $24,2 \%$ da produção mundial.

A laranja é cultivada em quase todos os estados brasileiros. Durante a produção de 1998 apenas os estados do Acre, Amapá, Rio Grande do Norte, Rondônia e Tocantins não se destacaram na produção nacional. Quanto à distribuição regional da área colhida, registram-se as seguintes porcentagens: Sudeste (81\%), Nordeste (11\%), Sul (5\%), Norte $(1,7 \%)$, e Centro-Oeste (menos de 1\%). A mesma classificação também pode ser aplicada quanto à produção em toneladas. Com relação aos estados maiores produtores destacam-se São Paulo (83\%), Bahia (3,8\%), Sergipe (3,3\%), Minas Gerais $(2,4 \%)$, Rio Grande do Sul (1,8\%), Pará (1,1\%), Paraná $(0,9 \%)$ e Santa Catarina $(0,7 \%)$. A produção restante (3\%) é originária dos demais estados (IBGE, 1998).

No Paraná, a tradicional região produtora de citros está localizada na Região do Alto Ribeira, principalmente no município de Cerro Azul. A produção em sua maior parte é de tangerinas, que atende basicamente aos mercados consumidores regionais de frutas frescas (STENZEL; CARVALHO, 1987).

A produção brasileira de suco de laranja concentrado congelado (SLCC) apresentou grande incremento na década de 70, devido principalmente aos preços internacionais atraentes. O suco de laranja concentrado congelado coloca-se como um dos principais produtos da pauta brasileira de exportações agrícolas (IAPAR, 1992; NEVES et al., 2001).

Segundo Koller (2001), os cítricos são as frutas mais consumidas pela população brasileira, quer como frutas frescas, quer como frutas industrializadas na forma de sucos, geléias, essências e outros. De acordo com dados da Companhia de Entrepostos e Armazéns Gerais de São Paulo Ceagesp - relativos aos anos 1990 e 1991 divulgados por Gonçalves e Souza, para cada $100 \mathrm{~kg}$ de frutas cítricas, apenas $25,3 \mathrm{~kg}$ de banana, $13,4 \mathrm{~kg}$ de maçã e 23,3 kg de mamão são comercializados. Em um levantamento em 1987-1988, concluiu que as populações de Criciúma-SC e Joinville-SC, consumiam respectivamente 14,5 e $20,2 \mathrm{~kg}$ de frutas cítricas/pessoa/ano.

Embora as indústrias de suco concentrado de laranja venham sendo acusadas de pagar preços bem inferiores aos praticados no mercado de frutas in natura, foi graças a elas que a citricultura paulista se transformou na potência que representa na atualidade. As indústrias ampliam as opções de venda para os produtores, pois absorvem frutos que seriam descartados no mercado in natura, por serem pequenos, excessivamente grandes ou apresentarem mau aspecto visual. Além disso, as indústrias geram empregos diretos e indiretos, geram divisas com as exportações e recolhem tributos (KOLLER, 2001).

Apesar do suco ser o principal produto da laranja, vários subprodutos com valor comercial são obtidos durante o seu processo de fabricação. Entre esses subprodutos estão os óleos essenciais (Safra 2000/01 - 21.867 t), d'limonene (Safra 2000/01 - 14.519 t) e o farelo de polpa cítrica (Safra 2000/01 - 769.731 t). Eles são usados para diferentes aplicações no mercado interno e externo, as quais incluem fabricação de produtos químicos e solventes, aromas e fragrâncias, tintas, cosméticos, suplemento para ração animal e outras (ABECITRUS, 2001).

A necessidade e a importância de caracterização do comércio de frutas em um grande centro consumidor, para auxiliar o entendimento de sua cadeia produtiva, podem ser exemplificadas por diversas pesquisas realizadas com frutas comercializadas em mercados atacadistas e varejistas de vários estados brasileiros (CARVALHO et al., 1992; FAGUNDES et al., 1999; FAGUNDES et al., 2000; FAGUNDES; YAMANISHI, 2001; FIORAVANÇO et al., 1994; PAIVA et al., 1996).

O objetivo deste trabalho foi observar a variação de preços no mercado varejista das frutas cítricas em Curitiba e Região Metropolitana no período de fevereiro de 1999 a janeiro de 2000. 


\section{Materiais e métodos}

Os preços praticados para as frutas cítricas foram obtidos através de visitas mensais em quatro mercados varejistas representativos da região de Curitiba e Região Metropolitana. Os preços reais foram corrigidos pelo INPC-IBGE para comparação entre os meses do ano, com base no mês de fevereiro de 1999 .

O delineamento experimental utilizado foi o de blocos ao acaso, com quatro repetições, sendo cada mercado uma repetição, e doze tratamentos, que foram as análises mensais efetuadas. A comparação estatística de preços mensais pelo teste de Tukey foi realizada somente quando havia oferta da fruta nos 12 meses do ano, como para a laranja 'Pêra' e a lima ácida 'Tahiti', ou apenas nos meses em que havia oferta devido à sazonalidade da produção, como para as tangerinas 'Murcote' (de fevereiro a dezembro) e 'Ponkan' (de março a agosto). Devido à ausência das laranjas 'Baia' e 'Lima', da lima 'Pérsia' e de outras cultivares de tangerinas em vários meses do ano na maior parte dos mercados analisados, a comparação estatística de preço destes cítricos não foi realizada.

\section{Resultados e discussão}

As cultivares de laranja comercializadas nos mercados de Curitiba e Região Metropolitana no período estudado foram a 'Baía', 'Pêra' e 'Lima'. A laranja 'Baía' foi a cultivar comercializada com maiores preços médios ( $\mathrm{R} \$ 1,18 / \mathrm{kg}$ ), porém com flutuações de preços ao longo do ano que variaram de $\mathrm{R} \$ 0,72 / \mathrm{kg}$ no mês de julho a $\mathrm{R} \$ 1,67 / \mathrm{kg}$ no mês de janeiro. A laranja 'Lima' foi comercializada com preço médio de $\mathrm{R} \$ 0,87 / \mathrm{kg}$, e teve seu menor e maior preço durante os meses de julho ( $R \$ 0,57 / \mathrm{kg}$ ) e fevereiro $(\mathrm{R} \$ 1,79 / \mathrm{kg})$, respectivamente. A cultivar 'Pêra' foi encontrada com os menores preços médios (R\$ $0,41 / \mathrm{kg}$ ) com variações de $\mathrm{R} \$ 0,25 / \mathrm{kg}$ no mês novembro a $\mathrm{R} \$ 0,65 /$ kg no mês de abril (Tabela 1).

O Paraná produz pequena porcentagem da produção nacional de laranjas, enquanto São Paulo detém a maior parte da produção brasileira (IBGE, 1998) e este fato representa uma forte influência da laranja paulista no mercado local. Além das épocas de colheita específicas para cada cultivar, outros fatores interferem na oferta e preço da fruta como a disponibilidade de fretes e a capacidade de estoque das frutas no local de comércio. As flutuações de preços para a laranja Baía de até $40 \%$ em relação à média anual, demonstram a influência destes fatores no preço final da fruta. A época de safra está diretamente relacionada a estas variações, uma vez que os menores preços ocorreram nos principais meses de colheita, representados pelo período de abril a agosto (KOLLER, 1994).

Variações de preços semelhantes ocorreram para as cultivares 'Lima' e 'Pera' para as quais nas épocas de safra (junho a novembro) ocorreram os menores preços, enquanto nos meses fora de safra foram registrados os maiores preços. Porém, estas variações foram mais intensas que as verificadas para a laranja 'Baía', uma vez que os preços variaram até $60 \%$ para a laranja 'Pêra' e até $107 \%$ para a laranja 'Lima'. O volume produzido destas cultivares, a eficiência na distribuição da fruta e a baixa capacidade de estoque dos mercados locais tornam mais evidentes estas variações muito dependentes da disponibilidade de entrega das frutas.

As cultivares de lima comercializadas foram a 'Tahiti' e a 'Pérsia', a preços médios de $\mathrm{R} \$ 0,78 / \mathrm{kg}$ e $\mathrm{R} \$ 1,37 / \mathrm{kg}$, respectivamente. As variações de preços da cultivar 'Tahiti' ao longo do ano foram de $\mathrm{R} \$ 0,43 / \mathrm{kg}$ (maio) a $\mathrm{R} \$ 1,48 / \mathrm{kg}$ (novembro) (Tabela 2). Esta grande variação de até $92 \%$ do mês de maior preço em relação ao preço médio anual demonstra também a forte influência da época de safra nos preços praticados. Conforme Koller (1994), a lima 'Tahiti' é colhida de fevereiro a maio, e sua capacidade de armazenamento pode variar de cinco semanas, até 12 semanas (CARVALHO, 1998; GAYET, 1995). Desta forma, mesmo com todos os cuidados de armazenagem, é pequena a capacidade de oferta da fruta após o mês de agosto, provocando elevação de preços nos mercados. Frutas oriundas de outras regiões do país poderiam interferir no preço praticado, porém não a ponto de torná-lo tão baixo quanto nas épocas de safra.

Para a cultivar 'Pérsia' as flutuações de preços ocorreram de $\mathrm{R} \$ 0,74 / \mathrm{kg}$ (agosto) a $\mathrm{R} \$ 1,82$ / kg (janeiro) (Tabela 2). Esta cultivar pode ser COlhida o ano todo em pomares domésticos, (MANICA, 1993) porém é pequena a importância de sua produção comercial. Isto faz com que seus preços sejam superiores aos praticados para a lima 'Tahi- 
ti' e, consequentemente, as variações anuais também sejam menores, em torno de $46 \%$ em relação à média anual. Mesmo nos tempos de estabilidade econômica no país, as variações de preços de frutas seguem uma dinâmica particular por serem produtos perecíveis.

As principais cultivares de tangerina comercializadas no período estudado foram a
'Ponkan' e a 'Murcote'. Outras cultivares como a 'Bravo', 'Paulista' e 'Rio' foram encontradas esporadicamente em menos de $25 \%$ das datas estudadas. Outras tangerinas, também com ocorrência menor que $25 \%$, estavam identificadas como os nomes comuns 'mexerica' e 'mimosa', sem possibilidade de distinção de cultivares.

TABELA 01 - Preços médios das laranjas 'Baía', 'Pera' e 'Lima' comercializadas no mercado varejista de Curitiba e Região Metropolitana de fevereiro de 1999 a janeiro de 2000.

Table 01 - Average prices of the baia, pêra and lima oranges commercialized in the retail market of Curitiba and in its Metropolitan Region from February of 1999 to January of 2000.

\begin{tabular}{|c|c|c|c|c|c|}
\hline \multirow[t]{2}{*}{ Meses } & \multicolumn{5}{|c|}{ Preço praticado $\left(\mathrm{R} \$ / \mathrm{kg}^{*}\right)$} \\
\hline & Baía & Lima & & Pêra* & $* *$ \\
\hline Fevereiro & 1,52 & 1,79 & & 0,53 & $\overline{a b c}$ \\
\hline Março & 1,04 & 0,97 & & 0,62 & ab \\
\hline Abril & 1,24 & 0,78 & & 0,65 & $\mathrm{a}$ \\
\hline Maio & 0,81 & 0,83 & & 0,40 & $\mathrm{bc}$ \\
\hline Junho & 1,06 & 0,66 & & 0,45 & $\mathrm{bc}$ \\
\hline Julho & 0,72 & 0,57 & & 0,43 & bc \\
\hline Agosto & 0,83 & 0,79 & & 0,36 & $\mathrm{c}$ \\
\hline Setembro & 1,12 & 0,90 & 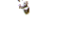 & 0,32 & $\mathrm{c}$ \\
\hline Outubro & 1,32 & 0,78 & & 0,26 & $\mathrm{c}$ \\
\hline Novembro & $* *$ & 0,78 & & 0,25 & $\mathrm{c}$ \\
\hline Dezembro & 1,66 & 0,88 & & 0,27 & bc \\
\hline Janeiro & 1,67 & 0,70 & & 0,39 & bc \\
\hline Média anual & 1,18 & 0,87 & & 0,41 & \\
\hline
\end{tabular}

* Preços corrigidos pelo INPC-IBGE com base no mês de fevereiro de 1999.

** Cultivar não encontrada no mês.

**** Médias seguidas por letras distintas diferem entre si pelo teste de Tukey ao nível de significância de 5 \%. 
TABELA 02 - Preços médio das lima, 'Tahiti' e 'Pérsia' comercializadas no mercado varejista de Curitiba e Região Metropolitana de fevereiro de 1999 a janeiro de 2000.

Table 02 - Average prices of the lima, tahiti and persia commercialized in the retail market of Curitiba and in its Metropolitan Region from February of 1999 to January of 2000.

\begin{tabular}{lccc}
\hline Meses & \multicolumn{4}{c}{ Preço praticado $\left(\mathrm{R} \$ / \mathrm{kg}^{*}\right)$} \\
\cline { 2 - 4 } & Pérsia & Tahiti*** \\
\hline Fevereiro & 1,70 & 0,46 & $\mathrm{~d}$ \\
Março & 1,58 & 0,52 & $\mathrm{~d}$ \\
Abril & 1,23 & 0,52 & $\mathrm{~d}$ \\
Maio & 1,54 & 0,43 & $\mathrm{~d}$ \\
Junho & 1,13 & 0,57 & $\mathrm{~d}$ \\
Julho & 1,27 & 0,53 & $\mathrm{~d}$ \\
Agosto & 0,74 & 1,13 & $\mathrm{bc}$ \\
Setembro & 1,18 & $1,32 \mathrm{ab}$ \\
Outubro & 1,04 & 0,92 & $\mathrm{c}$ \\
Novembro & $* *$ & $1,48 \mathrm{a}$ & \\
Dezembro & 1,80 & 0,98 & $\mathrm{bc}$ \\
Janeiro & 1,82 & 0,47 & $\mathrm{~d}$ \\
\hline Média anual & 1,37 & 0,78 & \\
\hline
\end{tabular}

* Preços corrigidos pelo INPC-IBGE com base no mês de fevereiro de 1999.

** Cultivar não encontrada no mês.

*** Médias seguidas por letras distintas diferem entre si pelo teste de Tukey ao nível de significância de $5 \%$.

A tangerina 'Murcote' foi comercializada com preços variando de $\mathrm{R} \$ 0,46 / \mathrm{kg}$ (julho) a $\mathrm{R} \$ 2,10 / \mathrm{kg}$ (fevereiro), determinado um preço médio anual de $\mathrm{R} \$ 1,19 / \mathrm{kg}$. O preço médio de comercialização para a cultivar 'Ponkan' foi de $\mathrm{R} \$ 0,56 / \mathrm{kg}$, com o maior preço encontrado de $\mathrm{R} \$ 1,18 / \mathrm{kg}$ (março) e o menor preço de $\mathrm{R} \$ 0,23$ / $\mathrm{kg}$ (junho) (Tabela 3).

As flutuações de preços das cultivares de tangerinas em relação ao preço médio anual atingiram percentuais de até $169 \%$ para a 'Murcote' e até $115 \%$ para a 'Ponkan'. Esta característica é influenciada pela época de colheita mais concentrada das frutas (de abril a julho para a 'Ponkan' e de julho a outubro para a 'Murcote') e menor capacidade de armazenamento, de até 6 semanas (CARVAlHO, 1998; COELHO, 1996). Estas características justificam o fato de, em muitos meses do ano, não haver oferta destas cultivares nos mercados. Por outro lado, quando há oferta de tangerinas nos mercados, os con- sumidores de frutas frescas têm demonstrado clara preferência pelas mesmas. Nos meses de junho a agosto, na média dos anos 1995-97, a quantidade de tangerinas comercializada através da Ceasa de São José (Santa Catarina), mesmo a preços bem superiores aos das laranjas, atingiu o mesmo volume de venda das laranjas, fato que se altera nos demais meses do ano, quando há menor produção de tangerinas, caindo a oferta. (KOLLER, 2001).

O grupo das outras cultivares ('Paulista', 'Bravo' e 'Rio') tiveram seu preço médio de $\mathrm{R} \$ 0,75 / \mathrm{kg}$, e seus maiores e menores preços foram encontrados nos meses de outubro ( $\mathrm{R} \$$ $0,93 / \mathrm{kg}$ ) e junho ( $\mathrm{R} \$ 0,41 / \mathrm{kg})$, respectivamente. O preço médio para as cultivares não identificadas foi de $\mathrm{R} \$ 1,29 / \mathrm{kg}$, com o maior preço encontrado em abril ( $\mathrm{R} \$ 3,04 / \mathrm{kg})$ e o menor preço em agosto ( $\mathrm{R} \$ 0,47 / \mathrm{kg}$ ). Algumas cultivares de tangerinas de importância nacional como a 'Cravo', 'Dancy', 'Satsuma', 'Mexerica-do-Rio', 'Mon- 
tenegrina' e 'Lee' não foram encontradas nos mercados avaliados. (COELHO, 1996)

A produção regionalizada de tangerinas na Região do Alto Ribeira, no município de Cerro Azul próximo à Curitiba/Paraná, para abastecimento do mercado de frutas frescas pode ser uma impor- tante causa do aparecimento de cultivares diversas e até mesmo sem identificação, caracterizando uma forma de mercado informal. (STENZEL; CARVALHO, 1997)

TABELA 03 - Preços médio das tangerinas 'Ponkan', 'Murcote', outras cultivares e não identificadas, comerciali-

zadas no mercado varejista de Curitiba e Região Metropolitana de fevereiro de 1999 a janeiro de 2000.

Table 03- Average prices of the tangerine ponkan, murcote and other cultivates including those not identified commercialized in the retail market of Curitiba and in its Metropolitan Region from February of 1999 to January of 2000.

\section{Considerações finais}

\begin{tabular}{lcrccc}
\hline Meses & \multicolumn{5}{c}{ Preço praticado $\left(\mathrm{R} \$ / \mathrm{kg}^{*}\right)$} \\
\cline { 2 - 6 } & Murcote*** & Ponkan & $\begin{array}{c}\text { Outras } \\
\text { cultivares }\end{array}$ & $\begin{array}{c}\text { Cultivares não } \\
\text { identificadas }\end{array}$ \\
\hline Fevereiro & $2,10 \mathrm{a}$ & $* *$ & $* *$ & $* *$ \\
Março & $1,91 \mathrm{ab}$ & $1,18 \mathrm{a}$ & 0,83 & 2,66 \\
Abril & $1,83 \mathrm{abc}$ & $1,05 \mathrm{a}$ & 0,76 & 3,04 \\
Maio & $1,67 \mathrm{abc}$ & $0,28 \mathrm{~b}$ & 0,65 & 0,71 \\
Junho & $1,60 \mathrm{abc}$ & 0,23 & $\mathrm{~b}$ & 0,41 & 0,76 \\
Julho & $0,46 \mathrm{c}$ & 0,28 & $\mathrm{~b}$ & 0,69 & 0,96 \\
Agosto & $0,54 \mathrm{c}$ & 0,32 & $\mathrm{~b}$ & 0,92 & 0,47 \\
Setembro & $0,55 \mathrm{c}$ & $* *$ & 0,83 & 0,86 \\
Outubro & 0,57 & $\mathrm{c}$ & $* *$ & 0,93 & 0,85 \\
Novembro & $0,85 \mathrm{bc}$ & $* *$ & $* *$ & $* *$ \\
Dezembro & $0,94 \mathrm{bc}$ & $* *$ & $* *$ & $* *$ \\
Janeiro & $* *$ & & $* *$ & $* *$ & $* *$ \\
\hline Média anual & 1,19 & 0,56 & 0,75 & 1,29 \\
\hline
\end{tabular}

* Preços corrigidos pelo INPC-IBGE com base no mês de fevereiro de 1999.

** Cultivar não encontrada no mês.

**** Médias seguidas por letras distintas diferem entre si pelo teste de Tukey ao nível de significância de $5 \%$. 
As cultivares de laranja comercializadas em Curitiba e Região Metropolitana foram 'Baia', 'Lima' e 'Pêra' com preços médios anuais de $\mathrm{R} \$ 1,18 / \mathrm{kg}$, $\mathrm{R} \$ 0,87 / \mathrm{kg}$ e $\mathrm{R} \$ 0,41 / \mathrm{kg}$, respectivamente.

As cultivares de lima comercializadas foram a 'Pérsia' e a 'Tahiti' a preços médios anuais de $\mathrm{R} \$ 1,37 / \mathrm{kg}$ e $\mathrm{R} \$ 0,78 / \mathrm{kg}$, respectivamente.

As principais cultivares de tangerinas comercializadas e identificadas foram a 'Murcote' e 'Ponkan' a preços médios anuais de $\mathrm{R} \$ 1,19 / \mathrm{kg}$ e $\mathrm{R} \$ 0,56 / \mathrm{kg}$, respectivamente.

Há cultivares de tangerinas comercializadas sem identificação.

\section{Referências}

ABECITRUS - Associação Brasileira dos Exportadores de Cítricos. Disponível: http:// www.abecitrus.com.br. Acessado em 26 nov. 2001.

CARVALHO, R.I.N. Conservação de frutas. In: CARVALHO, R.I.N. (Org.) Produção de frutas em pomar doméstico. Curitiba: Champagnat, 1998, p.141-153.

CARVALHO, R.I.N.; FIORAVANÇO, J.C.; PAIVA, M.C.; MANICA, I. Características físicas e químicas do mamão "papaya" comercializado em Porto Alegre. Rev. Bras. de Fruticult., Cruz das Almas, v. 14, n. 1, p.143-147, 1992.

COELHO, Y.S. Tangerina para exportação: aspectos técnicos da produção. Brasília: EmbrapaSPI, 1996. 42 p. (Série Publicações Técnicas FRUPEX; 24).

FAGUNDES, G.R.; YAMANISHI, O.K.; BORGO, L.A.; MANICA, I. Atributos de qualidade da banana 'Prata' comercializada entre setembro/97 e agosto/ 98, em 4 estabelecimentos de Brasília-DF. Rev. Bras. de Fruticult., Jaboticabal, v. 21, n. 3, p. 372374, dez. 1999.

FAGUNDES, G.R.; YAMANISHI, O.K.; MANICA, I.; LACERDA, C.S. Sazonalidade do abacaxi 'Pérola' nas CEASAs do Distrito Federal, São Paulo, Belo Horizonte e Rio de Janeiro, a partir do plano real. Rev. Bras. de Fruticult., Jaboticabal, v. 22, n. 2, p. 253-256, ago. 2000.

FAGUNDES, G.R.; YAMANISHI, O.K. Quantidade e preços da banana 'Prata' comercializada nas CEASAs do Distrito Federal, São Paulo, Belo Horizonte e Rio de Janeiro no período de 1995 a 1999. Rev. Bras. de Fruticult., Jaboticabal, v. 23, n. 3, p. 593-596, dez. 2001.

FIORAVANÇO, J.C.; PAIVA, M.F.; CARVALHO, R.I.N.; MANICA, I. Oferta de abacaxi nas CEASAs do Rio Grande do Sul, Santa Catarina e Paraná. Ciência Rural, Santa Maria, v. 24, n. 1, p. 73-76, 1994.

GAYET, J.P.; BLEINROTH, E.W.; MATALLO, M.; GARCIA, E.E.C.; GARCIA, A.E.; ARDITO, E.F.G.; BORDIN, M.R. Lima ácida 'Tahiti' para exportação: procedimentos de colheita e pós-colheita. Brasília: Embrapa-SPI, 1995. 36 p. (Série Publicações Técnicas FRUPEX; 12).

IAPAR- Instituto Agronômico do Paraná. A Citricultura no Paraná. Londrina-Pr: IAPAR 1992. 286 p.

IBGE. Anuário Estatístico do Brasil/Instituto Brasileiro de Geografia e Estatística. v. 58. p. 26, 1998.

KOLLER, O. L. Citricultura catarinense seus números e suas necessidades. Agropecuária Catarinense, v. 14, n. 1, p. 54-60, mar. 2001.

KOLLER, O. L. Citricultura: laranja, limão e tangerina. Porto Alegre: Ríger, 1994. 440 p.

MANICA, I. Planejamento do pomar doméstico. In: MANICA, I. (Coord.). Fruticultura em pomar doméstico: planejamento, formação e cuidados. Porto Alegre: Rígel, 1993. p. 30-57

NEVES, E.M.; DAYOUB, M.; DRAGONE, D.S.; NEVES, M.F. Citricultura brasileira: efeitos econômicos-financeiros, 1996 - 2000. Rev. Bras. de Fruticult., Jaboticabal, v. 23, n. 2, p. 432-436, ago. 2001.

PAIVA, M.C.; CARVALHO, R.I.N.; FIORAVANÇO, J.C. \& MANICA, I. Características da banana "nanicão" comercializada em Porto Alegre de outubro de 1991 a junho de 1992. Ciência e Agrotecnologia, Lavras, v. 20, n. 3, p. 275-278, jul/set. 1996.

SIMÃO, Salim. Tratado de Fruticultura. Piracicaba: FEALQ, 1998. 760 p.

STENZEL, N. M.; CARVALHO, S. L. C. Introdução. In Regionalização da Citricultura para o estado do Paraná. Londrina, PR: IAPAR - Fundação Instituto Agronômico do Paraná, 1997. p. 7. 